\title{
Fibrodisplasia osificante progresiva: informe de caso con diagnóstico temprano y propuesta de rehabilitación
}

Colmenares-Bonilla D ${ }^{1}$, González-Sandoval B²

\section{Resumen}

La fibrodisplasia osificante progresiva es una alteración genética extremadamente rara; se caracteriza por osificación heterotópica espontánea catastrófica, causada por un traumatismo mínimo y por malformación tipo hallux valgus en $95 \%$ de los casos. Su prevalencia es de 1 en 2,000,000 de habitantes. La mayoría de pacientes son mal diagnosticados en etapas tempranas y es frecuente que sean sometidos a pruebas diagnósticas causando discapacidad importante por limitación del movimiento.

En la primera década de vida hay episodios de dolor y edema en tejidos blandos, que precede a la osificación; éstos son precipitados por traumatismos mínimos, inyecciones intramusculares o procedimientos quirúrgicos, entre otras muchas causas. Las osificaciones causan dolor crónico, anquilosis articular, restricción de movimiento, infecciones graves, desnutrición y muerte por insuficiencia respiratoria. Generalmente, estos pacientes son sometidos a procedimientos diagnósticos o terapéuticos que agravan su problema.

Presentamos el caso de una paciente de 2.6 años de edad, proveniente de una comunidad rural, que fue diagnosticada tempranamente con este padecimiento y se describen propuestas del tratamiento de rehabilitación.

PALABRAS CLAVE: fibrodisplasia osificante progresiva, osificación heterotópica, rehabilitación.

\section{Fibrodysplasia Ossificans Progressiva. Case report with early diagnosis and Rehabilitation Proposal}

Colmenares-Bonilla D ${ }^{1}$, González-Sandoval B²

\footnotetext{
Abstract

Fibrodysplasia ossificans progressiva is an extremely rare genetic condition characterized by spontaneous catastrophic heterotopic
}

${ }^{1}$ Ortopedia Pediátrica, Hospital Regional de Alta Especialidad del Bajío.

${ }^{2}$ Medicina de Rehabilitación, Departamento de Medicina y Nutrición, Universidad de Guanajuato.

Recibido: 15 de abril del 2016

Aceptado: 1 de noviembre del 2016

Correspondencia

Beatriz González-Sandoval

bvgonzalez@ugto.mx

Este artículo debe citarse como

Colmenares-Bonilla D, González-Sandoval B. Fibrodisplasia osificante progresiva: informe de caso con diagnóstico temprano y propuesta de rehabilitación. Acta Pediatr Mex. 2017;38(2):101-107. DOI: http://dx.doi.org/10.18233/APM38No2pp101-1071361 
ossification secondary to the slightest trauma and associated hallux valgus malformation in $95 \%$ of cases. Its prevalence is $1: 2,000,000$ inhabitants. Most patients are misdiagnosed in early stages and are frequently subjected to diagnostic tests causing significant disability by limiting the movement. In the first decade of life, there are episodes of pain progressing to soft tissue edema, precipitated by minor trauma, intramuscular injections or surgical procedures among many other causes. The ossification leads to chronic pain, joint ankylosis, restriction of movement, serious infections, malnutrition and death from respiratory failure. They usually undergo diagnostic or therapeutic procedures that aggravate their condition. We report the case of a 2.6 year-old girl from a rural community who was precociously diagnosed with this condition, where proposals for rehabilitative treatment apply.

KEYWORDS: fibrodysplasia ossificans progressiva; heterotopic ossification; rehabilitation
${ }^{1}$ Ortopedia Pediátrica, Hospital Regional de Alta Especialidad del Bajío.

${ }^{2}$ Medicina de Rehabilitación, Departamento de Medicina y Nutrición, Universidad de Guanajuato.

Correspondence

Beatriz González-Sandoval bvgonzalez@ugto.mx

\section{INTRODUCCIÓN}

La fibrodisplasia osificante progresiva es un desorden genético extremadamente raro con herencia autosómica dominante; su frecuencia se ha calculado en 1 caso por cada 2 millones de nacidos vivos. ${ }^{1,2}$ La mayoría de los casos parecen ser mutaciones de novo y se considera como la alteración esquelética de osificación heterotópica más desastrosa y discapacitante. ${ }^{3}$ No hay predilección por sexo, etnia o situación geográfica. $^{1-3}$

La presentación clínica es diagnosticada con facilidad cuando se tienen en mente las características habituales de la enfermedad. ${ }^{4}$ El reto radica en el poco conocimiento y difusión de esta patología, lo que hace que el error en la detección ascienda hasta $80 \%$ de manera inicial ${ }^{5}$ retrasando el diagnóstico de fibrodisplasia osificante progresiva un promedio de cuatro años o más. ${ }^{1,3,6}$

Para el diagnóstico clínico basta la presencia de osificaciones heterotópicas cuyo patrón típico inicia en las regiones dorsal, axial, craneal y proximal, para más tarde involucrar las regiones ventral, apendicular, caudal y distal. Además de la malformación congénita del primer dedo del pie, variando del primer metatarso abducto, acortamiento, hallux valgus, varus o braquisindactilia. $^{6-8}$

Las osificaciones generalmente se inician en la primera década de la vida; son precipitadas por traumatismos menores ocasionando la aparición de un nódulo subcutáneo muy doloroso que evoluciona a inflamación, induración, dolor y, finalmente, osificación del tejido involucrando columna y esqueleto axial con resultado incapacitante. ${ }^{9-11}$

La calidad de vida se ve afectada severamente en el entorno personal, social y familiar, siendo la mayoría de los pacientes incapaces de auxiliarse para sus necesidades básicas conforme avanza el padecimiento. ${ }^{12}$ Durante las primeras manifestaciones hasta $67 \%$ de los pacientes son sometidos a estudios diagnósticos innecesarios que les conduce a la progresión de las osifica- 
ciones, daño y discapacidad permanente hasta en $50 \%$ de los casos. ${ }^{5}$

Dentro de los diagnósticos diferenciales se mencionan: malformaciones congénitas aisladas, braquisindactilia aislada, hallux valgus juvenil, sarcoma, tumor desmoideo, fibromatosis agresiva juvenil y linfedema. ${ }^{1}$

La importancia de presentar el caso actual radica en la difusión de la patología, donde su diagnóstico es sencillo si el clínico conoce de primera mano las características típicas y el ensombrecido pronóstico que causa la aceleración de la historia natural durante la búsqueda de una etiología de este padecimiento que semeja un problema tumoral.

Se sugieren medidas de rehabilitación, basadas en terapia ocupacional, con el objetivo de preservar la movilidad y disminuir la limitación de la función. A nuestro conocimiento, es de los reportes de caso con el paciente más joven y con menor tiempo desde la primera manifestación hasta el diagnóstico.

\section{CASO CLÍNICO}

Niña de 2.6 años de edad, originaria y residente de una comunidad rural, en condiciones de gran vulnerabilidad, con una hermana de seis años aparentemente sana; hijas de la misma madre con diabetes mellitus descontrolada de larga evolución y productos de diferente padre. La desintegración familiar hizo que la paciente se encuentre a cargo de la abuela materna.

En relación a la madre, se realizó exploración física sin encontrar datos sugestivos de la enfermedad en cuestión. Del padre se desconoce información y se desconoce de casos similares en la familia. En cuanto a su desarrollo psicomotor se encuentra sin alteraciones hasta referir padecimiento actual. La paciente se encontró dentro de la percentila 50 para peso y talla.

Historia de seis meses de caídas frecuentes con contusiones múltiples de cráneo y hombros. Las caídas causaron inflamación, dolor y posterior induración de la región frontal. Hubo limitación progresiva de la movilidad de la columna cervical y del miembro torácico derecho. En un centro de salud se realizó punción de un nódulo cervical, producto de una contusión, que evolucionó en unos días a una región indurada y luego pétrea con limitación posterior a la movilidad del hombro ipsilateral. Fue Ilevada a una unidad de rehabilitación para valoración por alteraciones en la marcha, lateralización de cuello e incapacidad de movimientos cervicales y de hombro derecho.

En la exploración física la paciente tuvo protuberancia frontal derecha evidente, de consistencia pétrea y no dolorosa. Postura con lateralización derecha de cabeza y cuello, proyección anterior del tronco, descenso de hombro y escápula. La marcha es independiente con claudicación derecha y limitación en la flexión y extensión de cadera que altera las fases de la marcha. Nulo braceo derecho (Figura 1).

La columna cervical tenía limitación de $70 \%$ de la movilidad en todos sus arcos sin lograr alineación a la posición neutra. En la cintura escapular se observaron protuberancias firmes en el borde lateral de ambas escápulas. Refería dolor en hombros al tratar de explorar arcos de movilidad por limitación articular (Figura 2). Miembros inferiores: protuberancias en cadera derecha que impiden la flexo-extensión; no se encontraron alteraciones en las rodillas con hallux valgus bilateral. Una serie ósea metastásica demostró osificación heterotópica en cráneo, hombros, escápulas, cadera derecha y se corroboró sinfalangismo en el primer dedo de ambos pies. 


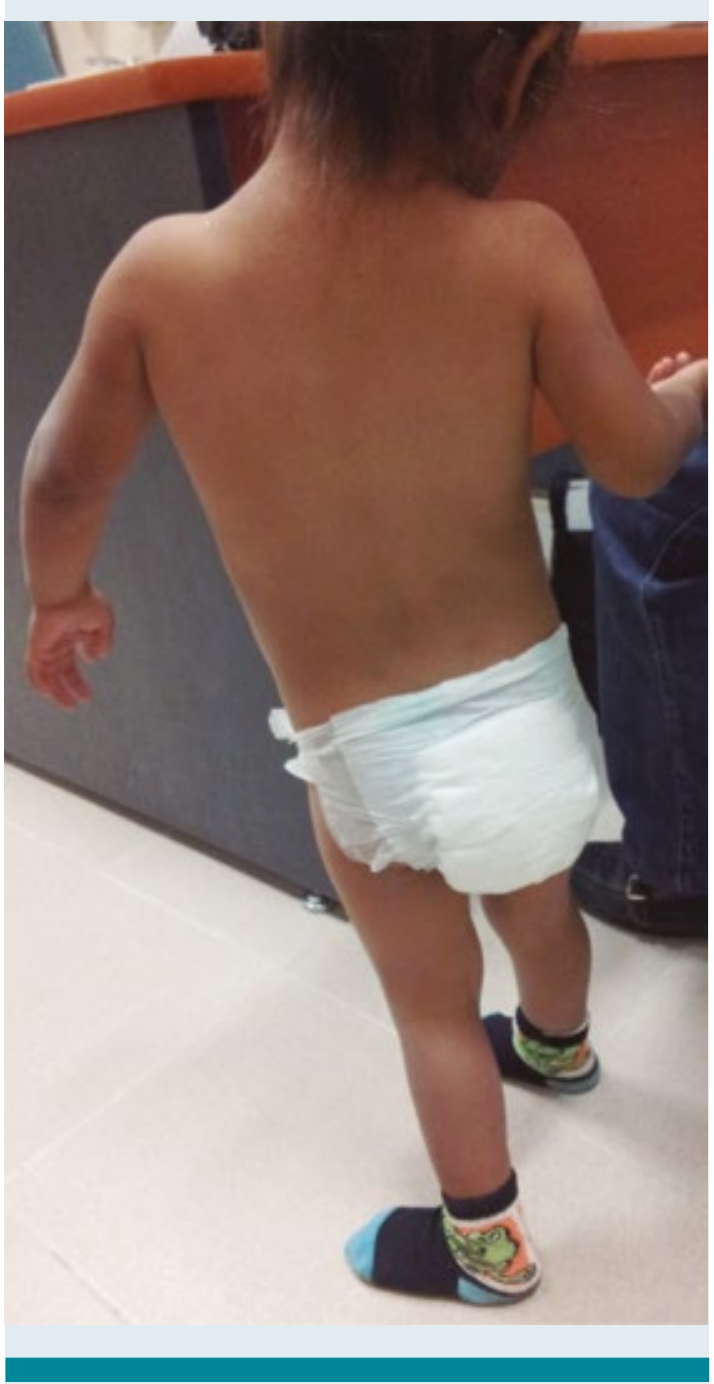

Figura 1. Aspecto clínico de la paciente a la primera cita. Nótese la limitación a la movilidad de columna cervical y lateralización del tronco. Asimismo, por pudor la mayor parte de estos pacientes no retiran inicialmente la calceta de sus pies.

La evolución de la paciente, desde el inicio de los signos clínicos, fue desfavorable y rápidamente progresiva debido a las múltiples lesiones resultado de caídas por la irregularidad del terreno, así como falta de adaptaciones en el hogar para la movilidad restringida que presentaba.

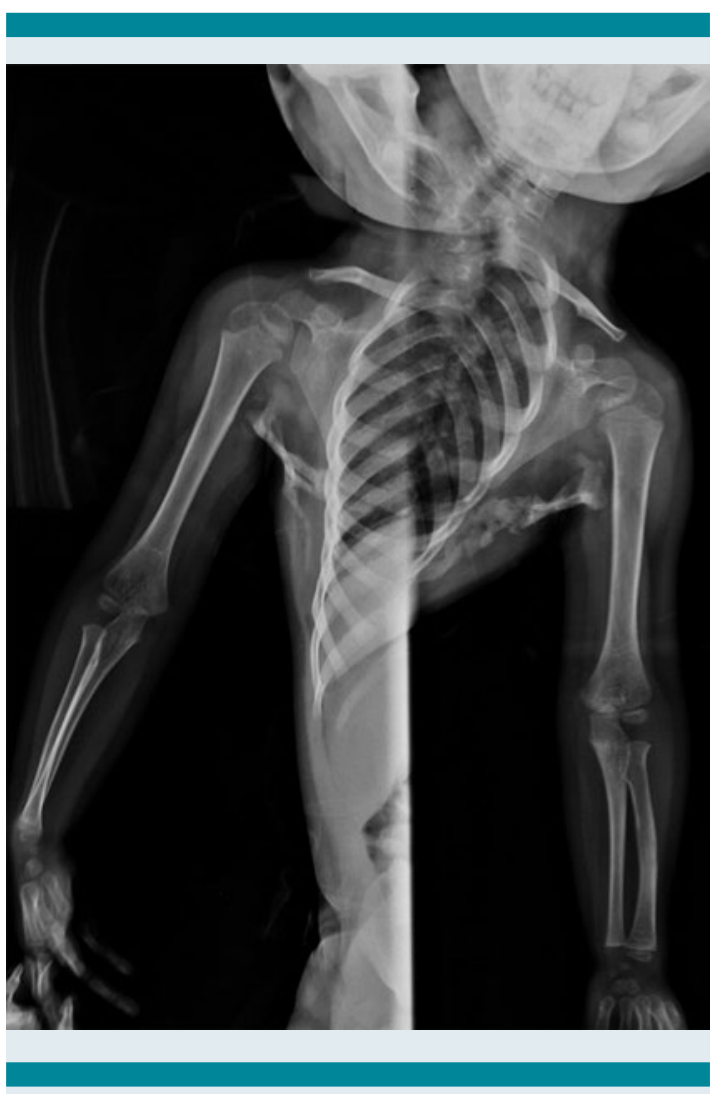

Figura 2. Puentes óseos formados desde el tercio proximal del humero hacia la caja torácica bilateral, también son observables los puentes en las apófisis espinosas de las vértebras cervicales formando una barra cervical.

Después de la valoración y el seguimiento de 24 meses no ha manifestado mayor cantidad de lesiones, ya que se lograron cambios adaptativos en su medio, así como información a los cuidadores primarios sobre la enfermedad.

\section{DISCUSIÓN}

La forma clásica de la fibrodisplasia osificante progresiva es fácilmente reconocible por las características clínicas y radiográficas, así como el antecedente de regiones del cuerpo que se induran y posteriormente se osifican, generalmente resultado de un traumatismo. 
Tres son las características distintivas de la fibrodisplasia osificante progresiva: 1) la alteración congénita del primer dedo del pie conocida como sinfalangismo que se da hasta en un 95\% de los pacientes (Figura 3); 2) la osificación heterotópica progresiva de tejidos blandos que se da usualmente en la primera década de la vida y es secundaria a traumatismos, inyecciones intramusculares, bloqueos mandibulares para procedimientos dentales, entre otras lesiones menores ${ }^{1,3,5}$ y 3 ) la formación progresiva de hueso alrededor del esqueleto axial, que se presenta aún sin condicionante traumático por osificación endocondral ${ }^{11}$ y que inicia generalmente en músculos paraespinales superiores, extendiéndose en sentido de axial a apendicular y de centro a la periferia. ${ }^{12,13}$

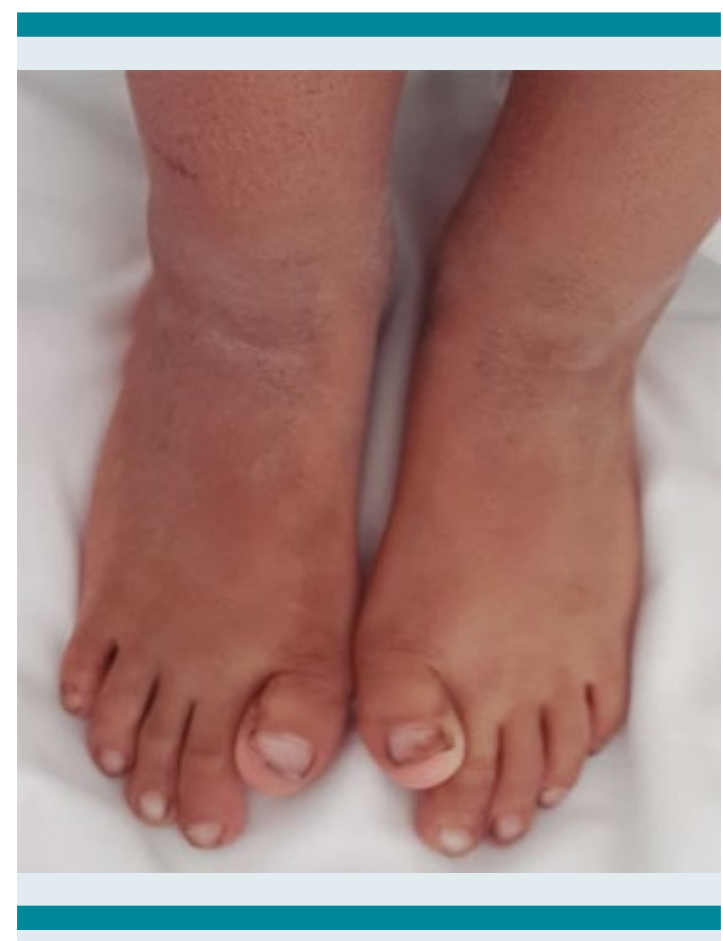

Figura 3. Deformidad característica del primer dedo de ambos pies de los pacientes con fibrodisplasia osificante progresiva Generalmente es mal diagnosticada como hallux valgus, braquisinfalangismo o braquisindactilia.
Debido a este patrón progresivo de osificación se forma un exoesqueleto que cubre al esqueleto axial e impide la movilidad a múltiples niveles en extremidades, caja torácica, columna y mandíbula. ${ }^{14-16}$ Es explicable que estos pacientes fallezcan como resultado de procesos infecciosos respiratorios, insuficiencia cardiaca, asfixia y desnutrición. $., 17,18$

La mutación genética causante de la fibrodisplasia osificante progresiva se ha descrito como una sustitución de un nucleótido único recurrente causando una mutación antisentido en el codón 206 (c.617G>A; R206H) en el dominio de activación de la glicina-serina del receptor del gen que codifica la activina IA (ACVR1), un receptor de proteína morfogenética ósea de tipo 1. 1,3-5,19 Se ha propuesto recientemente la posibilidad de un desorden inmunológico causante de esta cascada de osificación. ${ }^{11}$ Dicha sucesión de eventos se inicia posterior al traumatismo con inflamación seguida de una tumoración con edema doloroso, evoluciona en 9 a 12 semanas para desaparecer y organizarse en hueso laminar y puentes de hueso maduro que van progresando envolviendo fascias y vainas tendinosas. ${ }^{2,8-10,19}$

Las alteraciones radiográficas en la fibrodisplasia osificante progresiva avanzada incluyen cuerpos vertebrales altos con fusión de apófisis espinosas y fusión articular C2 a C7. Cuellos femorales anchos y cortos, así como osteocondromas en tibia medial y proximal. ${ }^{20}$ Ante sospecha de un diagnóstico temprano pueden ser útiles una tomografía y una resonancia magnética para detectar las lesiones. ${ }^{21}$

El error diagnóstico en pacientes con fibrodisplasia osificante progresiva puede ascender a $87 \%$ de los pacientes, independientemente de su origen étnico, antecedentes geográficos o especialidad médica. En un estudio realizado por Kitterman y sus colaboradores el diagnóstico erróneo más común fue el cáncer (32\%); el tiem- 
po medio desde el inicio de los síntomas hasta el diagnóstico correcto fue de 4.1 años y el número promedio de médicos consultados es de 6 antes del diagnóstico de fibrodisplasia osificante progresiva. ${ }^{5}$ En $67 \%$ de los pacientes se realizaron estudios invasivos innecesarios (biopsias), 68\% recibió tratamientos inadecuados. Hasta 49\% de los pacientes tuvieron pérdida permanente de la movilidad debido a intervenciones médicas invasivas que causaron osificación postraumática. ${ }^{5,12}$

Afortunadamente, nuestra paciente sólo fue sometida a un estudio invasivo, pero el diagnóstico certero se realizó después de seis meses de iniciados los síntomas.

Solamente existe un medicamento, en fase experimental, que puede auxiliar a estos pacientes: el palovaroteno, que actúa inhibiendo la progresión del tejido inflamado hacia una osificación heterotópica. La limitación con este fármaco, de reciente descripción, es que sólo actuará en la primera fase de la cascada de osificación, sin ser posible revertir las osificaciones ya presentes en el individuo. ${ }^{22}$

Actualmente, y sólo durante las primeras horas del traumatismo o lesión tisular, se ha demostrado la utilidad de altas dosis de corticosteroides para disminuir esta progresión, por lo que su uso se limita sólo a los brotes que afectan a las articulaciones principales, la mandíbula o la zona submandibular. ${ }^{13-15,23,24}$

El tratamiento de pacientes con fibrodisplasia osificante progresiva debe ser dirigido a la protección del niño, evitando cirugías, biopsias, anestesia local e inyecciones intramusculares. Las neumonías por restricción de la movilidad de la caja torácica deben ser tratadas enérgicamente pues son una amenaza de muerte. Los pacientes deben ser vacunados contra el virus de la influenza y el Haemophilus por vía intradérmica para evitar trauma. ${ }^{18,23}$
Debido a que nuestra paciente se encontraba en situación de gran vulnerabilidad social, económica, geográfica y pobre accesibilidad a los servicios de salud y de rehabilitación, se decidió realizar el análisis de su caso sugiriendo un programa de enseñanza que permitiera por lo menos evitar lesiones que propicien la osificación final. De esta forma se obtuvieron datos que nos permitieron conocer que en su entorno había grandes barreras arquitectónicas que daban lugar a caídas frecuentes: el piso irregular y de tierra, convivencia con animales de corral, el poco cuidado de la familia.

Se sugirió terapia ocupacional con actividades tendientes a mantener movilidad en columna cervical y extremidades, técnicas de protección articular, técnicas para mejorar el equilibrio y movilidad óculo cefálica en medida de lo posible, evaluación de ambiente familiar y escolar diseñando adecuaciones y ayudas técnicas para a disminuir o prevenir el daño.

La incoordinación de la marcha de estos niños causada por la malformación de los pies, la rigidez progresiva y la incapacidad de movimientos cervicales, predispone a caídas y mayores traumatismos frecuentes y más severos que la población en general. Se deben evitar las actividades de alto riesgo y asegurar los movimientos en el ambiente familiar y de rutina. ${ }^{12,23}$

\section{REFERENCIAS}

1. Kaplan FS, Xu M, Glasser DL, Collins F, Connor M, Kitterman $\mathrm{J}$, et al. Early Diagnosis of Fibrodysplasia Ossificans Progressiva. Pediatrics. 2008;121:e1295-e1300.

2. Delataycki M, Rogers JG. The Genetic of Fibrodysplasia Ossificans Progressiva. Clin Orthop Relat Res. 1998;346:15-18.

3. Kaplan FS, Le Merrer M. Fibrodysplasia ossificans progressive. Best Pract Res Clin Rheumatol. 2008;22(1):191-205.

4. Pignolo RJ, Shore EM, Kaplan FS. Fibrodysplasia Ossificans Progressiva: Diagnosis, Management, and Therapeutic Horizons. Pediatric endocrinology reviews : PER. 2013;10(0 2):437-48. 
5. Kitterman JA, Kantanie S, Rocke DM, Kaplan FS. latrogenic Harm Caused by Diagnostic Errors in Fibrodysplasia Ossificans Progressiva. Pediatrics. 2005;116 (5):e654-e661.

6. Al Kaissi A, Kenis V, Ben Ghachem M, et al. The Diversity of the Clinical Phenotypes in Patients With Fibrodysplasia Ossificans Progressiva. Journal of Clinical Medicine Research. 2016;8(3):246-53.

7. Kaplan FS, Glaser DL, Shore EM, Deirmengian GK, Gupta R, Delai $\mathrm{P}$, et al. The Phenotype of Fibrodysplasia Ossificans Progressiva. Clin Rev Bone Miner Metab. 2005;3-3:183-188.

8. Pignolo RJ, Shore EM, Kaplan FS. Fibrodysplasia Ossificans Progressiva: Clinical and Genetic Aspects. Orphanet J Rare Dis. 2011;6:80. doi: 10.1186/1750-1172-6-80.

9. Rogoveanu O, Traistaru R, Streba C, Stoica Z, Popescu R. Clinical, evolution and therapeutical considerations upon a case of fibrodysplasia ossificans progressiva (FOP). Journal of Medicine and Life. 2013;6(4):454-58.

10. Kaplan FS, Chakkalakal SA, Shore EM. Fibrodysplasia ossificans progressiva: mechanisms and models of skeletal metamorphosis. Disease Models \& Mechanisms. 2012;5(6):756-62.

11. Pignolo RJ, Bedford-Gay C, Liljesthröm M, Durbin-Johnson $B P$, Shore EM, Rocke DM, Kaplan FS. The Natural History of Flare-Ups in Fibrodysplasia Ossificans Progressiva (FOP): A Comprehensive Global Assessment. J Bone Miner Res. 2016;31(3):650-6.

12. Ortiz-Agapito F, Colmenares-Bonilla D. Quality of life of patients with fibrodysplasia ossificans progressiva. Journal of Children's Orthopaedics. 2015;9(6):489-93.

13. Kocyigit H, Hizli N, Memis A, Sabah D. A Severely Disabling Disorder: Fibrodysplasia Ossificans Progressiva. Clin Rheumatol. 2001;20:273-75.

14. Mahboubi S, Glaser DL, Shore EM, Kaplan FS. Fibrodysplasia ossificans progressive. Pediatr Radiol. 2001;31:307-14.

15. Mishima K, Kitoh H, Haga N, et al. Radiographic characteristics of the hand and cervical spine in fibrodysplasia ossificans progressiva. Intractable \& Rare Diseases Research. 2014;3(2):46-51.
16. Verma AK, Aga P, Singh SK, Singh R. The stone man disease: fibrodysplasia ossificans progressiva: imaging revisited. BMJ Case Reports. 2012;2012:bcr2012006422. doi:10.1136/bcr-2012-006422.

17. Moore RE, Dormans JP, Drummond DS, Shore EM, Kaplan FS, Auerbach JD. Chin on Chest Deformity in Patients with Fibrodysplasia Ossificans Progressiva. J Bone Joint Surg Am. 2009;91:1497-502.

18. Kaplan FS, Zasloff MA, Kitterman JA, Shore EM, Hong CC, Rocke DM. Early Mortality and Cardiorespiratory Failure in Patients with Fibrodysplasia Ossificans Progressiva. The Journal of Bone and Joint Surgery American volume. 2010;92(3):686-91.

19. Kaplan FS, Xu M, Seemann P, et al. Classic and Atypical FOP Phenotypes are Caused by Mutations in the BMP Type I Receptor ACVR1. Human mutation. 2009;30(3):379-90.

20. Deirmengian GK, Hebela NM, O'Connell M, Glaser DL, Shore EM, Kaplan FS. Proximal Tibial Osteochondromas in Patients with Fibrodysplasia Ossificans Progressiva. The Journal of Bone and Joint Surgery American volume. 2008;90(2):366-74.

21. Tulchinsky M. Diagnostic Features of Fibrodyslpasia (Myositis) Ossificans Progressiva on Bone Scan. Clin Nucl Med. 2007;32:616-19.

22. Salin A Chakkalakal, Kenta Uchibe, Michael R Convente, Deyu Zhang, Aris N Economides, Frederick S Kaplan, et al. Palovarotene Inhibits Heterotopic Ossification and Maintains Limb Mobility and Growth in Mice With the HumanACVR1R206H Fibrodysplasia Ossificans Progressiva (FOP) Mutation. Journal of Bone and Mineral Research, 2016; DOI: 10.1002/jbmr.2820.

23. Kaplan FS, Shore EM, Pignolo RJ. The medical management of fibrodysplasia ossificans progressiva: current treatment considerations. Clin Proc Intl Clin Consort FOP. 2011:4;1-100.

24. Brantus JF, Meunier PJ. Effects of Intravenous Etidronate and Oral Corticosteroids in Fibrodysplasia Ossificans Progressiva. Clin Orthop Relat Res.1998;346:117-120. 